\title{
Morphology Transformation of Giant Vesicles by a Polyelectrolyte for an Artificial Model of a Membrane Protein for Endocytosis
}

\author{
Eri Yoshida \\ Department of Environmental and Life Sciences, Toyohashi University of Technology, Toyohashi, Japan
}

Email address:

eyoshida@ens.tut.ac.jp

\section{To cite this article:}

Eri Yoshida. Morphology Transformation of Giant Vesicles by a Polyelectrolyte for an Artificial Model of a Membrane Protein for Endocytosis. Colloid and Surface Science. Vol. 3, No. 1, 2018, pp. 6-11. doi: 10.11648/j.css.20180301.12

Received: February 20, 2018; Accepted: March 6, 2018; Published: March 23, 2018

\begin{abstract}
The morphology transformation of giant vesicles consisting of amphiphilic poly(methacrylic acid)-blockpoly(methyl methacrylate-random-methacrylic acid-random-3-sulfopropyl methacrylate potassium salt), PMAA- $b$-P(MMA-rMAA- $r$-SpMA), was investigated using poly(allylamine hydrochloride) (PAH) as an artificial model of a membrane protein for endocytosis. The polymerization-induced self-assembly of the PMAA- $b$-P(MMA- $r$-MAA- $r$-SpMA) using the nitroxidemediated photo-controlled/living radical polymerization technique produced spherical vesicles in the absence of PAH, while it provided a fused sheet in its presence at a 1.0 molar ratio of the allylamine hydrochloride unit (AH) to the SpMA unit. It was suggested that the PAH connected the SpMA units by an electrostatic interaction. The fused sheet changed into combined vesicles as the $\mathrm{AH} / \mathrm{SpMA}$ ratio increased, and at $\mathrm{AH} / \mathrm{SpMA}=10.0$, the morphology was transformed into spherical vesicles much smaller than the original vesicles. The morphology transformation by soaking the original spherical vesicles in a PAH solution demonstrated that the PAH caused the division of the vesicles into much smaller spherical vesicles.
\end{abstract}

Keywords: Giant Vesicles, Amphiphilic Diblock Copolymer, Photo NMP-Induced Self-assembly, Morphology Transformation, Vesicle Division, Polyelectrolyte, Electrostatic Interaction

\section{Introduction}

Vesicles in vivo are involved in many important processes essential to maintaining vital activity, such as metabolism [1, 2], material transport and storage [3], and signal transmission and transduction [4]. The vesicles are formed during cytosis by budding separation from the mother biomembrane $[5,6]$. Endocytosis is a vesicle-mediated transport initiated by plasma membrane invagination [7, 8]. Potocytosis is the endocytosis mediated by caveolae vesicles formed out of caveolae, the flask-shaped membrane invaginations [9-11]. Caveolin-1 is an integral membrane protein for the caveolae membrane and is involved in the caveolar formation $[12,13]$. Caveolin-1 has a structure consisting of three distinct regions; i.e., an amino-terminal domain, hairpin loop region, and carboxy-terminal domain. Both terminal domains are oriented towards the cytosolic face of the plasma membrane, while the hairpin loop region is inserted within the hydrophobic phase of the membrane bilayer [14].

Giant vesicles consisting of an amphiphilic poly (methacrylic acid)-block-poly (methyl methacrylate-randommethacrylic acid) diblock copolymer, PMAA- $b$-P(MMA- $r$ MAA), are an artificial model of the biomembrane for cells and organelles based on the similarities in their structure [15], stimuli-responsive behavior [16, 17], and membrane permeability [18]. Various morphologies have been found for the diblock copolymer depending on the block length [19, 20], types of the monomer units [21-23], insertion of the segment copolymer [24], and conditions of the vesicle preparation [25] by the polymerization-induced selfassembly using the nitroxide-mediated photo controlled/living radical polymerization (photo NMP) technique [26]. The morphologies include spherical vesicles [15], the elliptical [25], worm-like [19, 20, 27], cup-like [27], key-shaped [19], villus-like [28], and anastomosed tubular networks continuing with a fenestrated sheet [17]. Furthermore, the vesicles supporting the ionic monomer units of the 3-sulfopropyl methacrylate potassium salt (SpMA) on the hydrophobic block enhanced the permeability of the vesicles for ionic compounds, such as Rhodamine B, based 
on the electrostatic interaction in the hydrophobic phase [18].

This paper describes the morphology changes in the vesicles consisting of PMAA- $b$-P(MMA-r-MAA- $r$-SpMA) using poly (allylamine hydrochloride) (PAH) as an artificial model of caveolin-1. PAH has been reported to interact with poly (methacrylic acid) (PMAA) to produce multilayer complexes [29]. This polyelectrolyte also interacted with ionic compounds with low molecular weights to produce micellar aggregates in water $[30,31]$. It is expected that the morphology of the vesicles is dominated by PAH because this polyelectrolyte undergoes an interaction with the PMAA blocks in the hydrophilic surface of the vesicles and simultaneously with the SpMA units in their hydrophobic phases.

\section{Experimental Work}

\subsection{Instrumentation}

The polymerization-induced self-assembly through the photo NMP was performed using an Ushio optical modulex BA-H502, an illuminator OPM2-502H with a highillumination lens UI-OP2SL, and a 500W super highpressure UV lamp (USH-500SC2, Ushio Co. Ltd.). ${ }^{1} \mathrm{H}$ NMR measurements were conducted using Jeol ECS400 and ECS500 FT NMR spectrometers. Gel permeation chromatography (GPC) was performed at $40^{\circ} \mathrm{C}$ using a Tosoh GPC-8020 instrument equipped with a DP-8020 dual pump, a CO-8020 column oven, and an RI-8020 refractometer. Two gel columns, Tosoh TSK-GEL $\alpha-\mathrm{M}$ were used with $N, N$ dimethylformamide (DMF) containing $30 \mathrm{mM} \mathrm{LiBr}$ and 60 $\mathrm{mM} \mathrm{H}_{3} \mathrm{PO}_{4}$ as the eluent. Field emission scanning electron microscopy (FE-SEM) measurements were performed using a Hitachi SU8000 scanning electron microscope.

\subsection{Materials}

Methacrylic acid (MAA) was purified by distillation under reduced pressure. Methyl methacrylate (MMA) was washed with $5 \% \mathrm{NaOH}$ aqueous solution and water. After dried with anhydrous $\mathrm{MgSO}_{4}$, the MMA was distilled over calcium hydride. The purified MMA was degassed with Ar for $15 \mathrm{~min}$ with stirring just before use. 4-Methoxy-2,2,6,6tetramethylpiperidine-1-oxyl (MTEMPO) was prepared as reported previously [32]. 2,2'-Azobis[2-(2-imidazolin-2yl)propane] (V-61) was purchased from Wako Pure Chemical Industries. PAHs with $M n=150,000,15,000$, and 3,000 were obtained from Nittobo. These chemicals were used without further purification. (4-tert-Butylphenyl)diphenyl-sulfonium triflate $\left({ }^{t} \mathrm{BuS}\right)$ and SpMA were purchased from SigmaAldrich and used as received. Methanol (MeOH) was refluxed over magnesium with a small amount of iodine for several hours and then distilled. Distilled water was purchased from Wako Pure Chemical Industries and purified by distillation. Extremely pure $\mathrm{N}_{2}$ gas with over 99.9995 vol\% purity and Ar gas with over 99.999 vol\% purity were purchased from Taiyo Nippon Sanso Corporation.

\subsection{Preparation of PMAA End-Capped with MTEMPO}

PMAA end-capped with MTEMPO was prepared as reported previously [19]; V-61 (22.8 mg, $0.0911 \mathrm{mmol})$, MTEMPO (18 mg, $0.0966 \mathrm{mmol}),{ }^{t} \mathrm{BuS}(24.0 \mathrm{mg}, 0.0512$ mmol), MAA (2.030 g, $23.6 \mathrm{mmol})$, and $\mathrm{MeOH}$ (4 mL) were placed in a $30-\mathrm{mL}$ test tube joined to a high vacuum valve. The contents were degassed several times using a freezepump-thaw cycle and then charged with $\mathrm{N}_{2}$. The photo NMP was carried out at room temperature for $5.5 \mathrm{~h}$ with irradiation at 8.9 amperes by a reflective light using a mirror with a $500 \mathrm{~W}$ super high-pressure UV lamp in order to avoid any thermal polymerization caused by the direct irradiation [33]. $\mathrm{MeOH}(3.5 \mathrm{~mL})$ and distilled water $(2.5 \mathrm{~mL})$ degassed by bubbling Ar for 15 min were added to the product under a flow of Ar. After the product was completely dissolved in the aqueous $\mathrm{MeOH}$ solution, part of the mixture (ca. $0.5 \mathrm{~mL}$ ) was withdrawn using a syringe to determine the conversion of MAA and molecular weight of the PMAA. The monomer conversion $(71 \%)$ was determined by ${ }^{1} \mathrm{H}$ NMR, while the molecular weight $(M n=16,600)$ and molecular weight distribution $(M w / M n=1.57)$ were estimated by GPC based on PMAA standards. The degree of polymerization (DP = 189) was calculated on the basis of the molecular weights of the PMAA, the initiator fragment, and MTEMPO. The concentration of the PMAA was determined to be $9.66 \mathrm{mM}$ based on the initial concentration of MTEMPO by a disregard for a slight contraction in volume due to the mixing of the methanol and water. The solution withdrawn was poured into ether $(50 \mathrm{~mL})$ to precipitate the PMAA. The precipitate was collected by filtration and dried in vacuo for several hours to obtain the PMAA end-capped with MTEMPO (32.1 mg).

\subsection{Photo NMP-Induced Self-Assembly for PMAA-b-P(MMA-r-MAA-r-SpMA) in the Presence of PAH. General Procedure}

PAH $(\mathrm{Mn}=150,000)(61.8 \mathrm{mg}, 0.661 \mathrm{mmol}$ as the allylamine hydrochloride (AH) unit) was dissolved in an aqueous $\mathrm{MeOH}$ solution $\left(6.4 \mathrm{~mL}, \mathrm{MeOH} / \mathrm{H}_{2} \mathrm{O}=3 / 1 v / v\right)$. The MTEMPO-capped PMAA solution containing unreacted MAA due to a $71 \%$ MAA conversion $(2 \mathrm{~mL}$ containing $0.0193 \mathrm{mmol}$ of the PMAA and $1.35 \mathrm{mmol}$ of the unreacted MAA), MMA (664.6 mg, $6.64 \mathrm{mmol})$, MAA (23.3 mg, 0.271 mmol), SpMA (5.1 mg, $0.0207 \mathrm{mmol})$, the PAH solution (1 $\mathrm{mL}$ containing $9.66 \mathrm{mg}$ of $\mathrm{PAH}, 0.103 \mathrm{mmol}$ as the $\mathrm{AH}$ unit), and an aqueous $\mathrm{MeOH}$ solution $\left(1 \mathrm{~mL}, \mathrm{MeOH} / \mathrm{H}_{2} \mathrm{O}=3 / 1\right.$ $v / v$ ) were placed in a $30-\mathrm{mL}$ test tube joined to a high vacuum valve under a flow of Ar. The initial molar ratio of the monomers was MMA/MAA/SpMA = $0.8018 / 0.1957 / 0.0025$. The contents were degassed several times using a freeze-pump-thaw cycle and finally charged with $\mathrm{N}_{2}$. The polymerization was carried out for $7 \mathrm{~h}$ at room temperature with a 600 -rpm stirring speed by irradiation at 8.9 amperes using a reflective light from a mirror with a 500W super high-pressure UV lamp. After the polymerization, part of the resulting dispersion solution (ca. 
$0.2 \mathrm{~mL}$ ) was withdrawn using a syringe to determine the monomer conversions. An aqueous $\mathrm{MeOH}$ solution $(20 \mathrm{~mL}$, $\mathrm{MeOH} / \mathrm{H}_{2} \mathrm{O}=3 / 1 v / v$ ) was added to the dispersion solution to precipitate vesicles. The vesicles were cleaned with the
$\mathrm{MeOH}$ solution by a repeated sedimentation-redispersion process. The resulting vesicles were stored in the presence of a small amount of the $\mathrm{MeOH}$ solution.

Table 1. The PMAA-b-P(MMA-r-MAA-r-SpMA) produced by the photo NMP-induced self-assembly.

\begin{tabular}{|c|c|c|c|c|c|c|c|c|c|c|c|}
\hline \multirow{2}{*}{$\begin{array}{l}\text { DP of } \\
\text { PMAA }\end{array}$} & \multicolumn{2}{|l|}{ PAH } & \multicolumn{3}{|c|}{ Conversion (\%) } & \multicolumn{3}{|c|}{ Molar ratio of unit ${ }^{\mathrm{a}}$} & \multirow{2}{*}{$\mathbf{D P}^{\mathrm{a}}$} & \multirow{2}{*}{$M n^{\mathrm{b}}$} & \multirow{2}{*}{$M w / M n^{\mathrm{b}}$} \\
\hline & $M n$ & AH/SpMA & ММА & MAA & SpMA & МMA & MAA & SpMA & & & \\
\hline 193 & 150,000 & $\infty^{c}$ & 70 & 50 & - & 0.850 & 0.150 & - & 283 & 48,200 & 1.59 \\
\hline 193 & 150,000 & 0.5 & 70 & 50 & 70 & 0.850 & 0.147 & 0.003 & 284 & 49,800 & 1.59 \\
\hline 189 & 150,000 & 1.0 & 73 & 56 & 75 & 0.839 & 0.158 & 0.003 & 299 & 47,600 & 1.61 \\
\hline 189 & 150,000 & 2.5 & 67 & 48 & 60 & 0.849 & 0.149 & 0.002 & 270 & 48,100 & 1.62 \\
\hline 189 & 150,000 & 5.0 & 71 & 50 & 79 & 0.852 & 0.145 & 0.003 & 287 & 51,900 & 1.61 \\
\hline 189 & 150,000 & 10.0 & 72 & 55 & 70 & 0.840 & 0.157 & 0.003 & 292 & 52,300 & 1.63 \\
\hline 188 & 15,000 & 1.0 & 64 & 44 & 60 & 0.853 & 0.145 & 0.002 & 258 & 41,300 & 1.63 \\
\hline 188 & 3,000 & 1.0 & 80 & 61 & 76 & 0.840 & 0.158 & 0.002 & 327 & 43,900 & 1.56 \\
\hline
\end{tabular}

$[\mathrm{MMA}]_{0}=1.66 \mathrm{M},[\mathrm{MAA}]_{0}=0.406 \mathrm{M},[\mathrm{SpMA}]_{0}=5.18 \mathrm{mM}$

${ }^{\text {a }}$ For the P(MMA-r-MAA- $r$-SpMA) block

${ }^{\mathrm{b}}$ Estimated by GPC based on PMAA standards for PMAA- $b$-P(MMA-r-MAA- $r$-SpMA).

${ }^{\mathrm{c}}[\mathrm{AH}]_{0}=5.20 \mathrm{mM}$, equal to the concentration for the SpMA-containing copolymer at $\mathrm{AH} / \mathrm{SpMA}=1$.

${ }^{\mathrm{d}}[\mathrm{MAA}]_{0}=0.391 \mathrm{M},[\mathrm{SpMA}]_{0}=20.7 \mathrm{mM}$

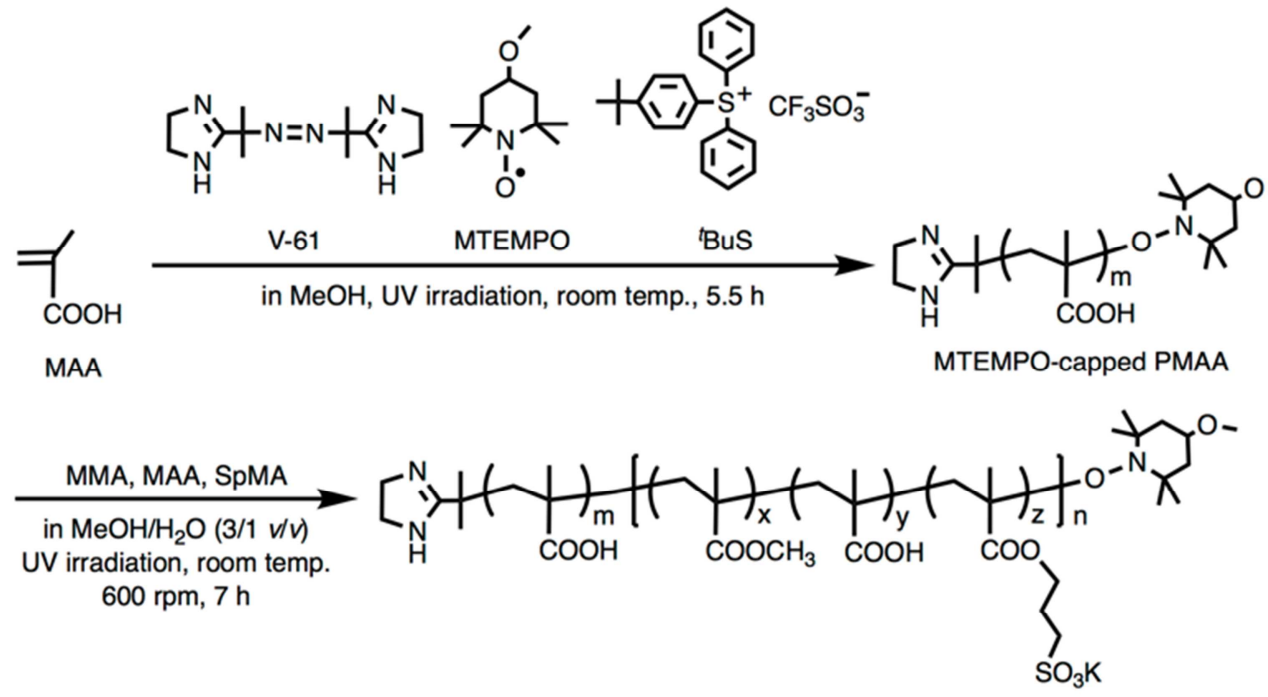

PMAA- $b$-P(MMA-r-MAA- $r$-SpMA)

Figure 1. Synthesis of PMMA-b-P(MMA-r-MAA-r-SpMA).

\subsection{Soaking of the Spherical Vesicles in a PAH Solution}

PAH (5.2 mg, $0.0556 \mathrm{mmol}$ as the $\mathrm{AH}$ unit) was dissolved in an aqueous $\mathrm{MeOH}$ solution $\left(10 \mathrm{~mL}, \mathrm{MeOH} / \mathrm{H}_{2} \mathrm{O}=3 / 1\right.$ $v / v)$. The vesicles $(D n=2.37 \mu \mathrm{m}, D w / D n=2.12)[18]$ consisting of $\mathrm{PMAA}_{216}-b-\mathrm{P}\left(\mathrm{MMA}_{0.830^{-}} r-\mathrm{MAA}_{0.168}-r-\right.$ $\left.\mathrm{SpMA}_{0.002}\right)_{321}\left(43.7 \mathrm{mg}\right.$ containing $5.57 \times 10^{-4} \mathrm{mmol}$ as the SpMA unit) were dispersed in an aqueous $\mathrm{MeOH}$ solution $\left(9.5 \mathrm{~mL}, \mathrm{MeOH} / \mathrm{H}_{2} \mathrm{O}=3 / 1 v / v\right)$. The PAH solution $(0.5 \mathrm{~mL}$, $2.78 \times 10^{-3} \mathrm{mmol}$ as the $\mathrm{AH}$ unit $)(\mathrm{AH} / \mathrm{SpMA}=5.0)$ was added to the dispersion at room temperature. The mixture was vigorously shaken and stored at room temperature for 117 days to precipitate the vesicles. The precipitates were isolated by decantation and dried in air to subject to FESEM. For a control experiment, the vesicles $(D n=1.70 \mu \mathrm{m}$, $D w / D n=1.56)$ consisting of $\mathrm{PMAA}_{216}-b-\mathrm{P}\left(\mathrm{MMA}_{0.829}-r-\right.$ $\left.\mathrm{MAA}_{0.171}\right)_{318}[18]$ were subjected to the soaking.

\subsection{SEM Observations}

The aggregates obtained by the photo NMP-induced selfassembly and the reaction of the vesicles with $\mathrm{PAH}$ were dried in air and subjected to the FE-SEM measurements. The morphology and vesicular size were determined by the FESEM observation at $1.0 \mathrm{kV}$ without coating. The size distribution of the spherical vesicles was estimated as reported previously [34].

\section{Results and Discussion}

In order to evaluate the effect of PAH on the morphology of the vesicles, the polymerization-induced self-assembly was performed for PMAA- $b$-P(MMA-r-MAA- $r$-SpMA) in the presence of $\mathrm{PAH}$ using the photo NMP technique. The 
random block copolymerization of MMA, MAA, and SpMA was carried out using PMAA end-capped with MTEMPO by $\mathrm{UV}$ irradiation at room temperature in an aqueous $\mathrm{MeOH}$ solution (Figure 1). The resulting diblock copolymers are listed in Table 1. The monomer conversions and the molar ratio of the unit in the P(MMA-r-MAA-r-SpMA) block were determined by ${ }^{1} \mathrm{H}$ NMR [18]. The copolymers contained the MMA units of $0.84 \sim 0.85$ in the hydrophobic P(MMA- $r$ MAA- $r$-SpMA) block.

FE-SEM images of the morphologies formed by the diblock copolymers are shown in Figure 2. The PMAA- $b$ P(MMA-r-MAA) diblock copolymer without SpMA units produced spherical vesicles in the presence of PAH. The diameter and distribution of the vesicles were $D n=1.51 \mu \mathrm{m}$ and $D w / D n=2.15$, respectively. Compared to the vesicles $(D n=1.70 \mu \mathrm{m}, D w / D n=1.56)$ formed in the absence of PAH [18], the copolymer provided smaller vesicles with a broader distribution in the presence of PAH. The interaction of the PMAA block with the more hydrophilic PAH expands the hydrophilic surface area in the critical packing shape of the copolymer, resulting in the formation of smaller vesicles with a higher curvature (Figure 3). However, the PAH did not cause any intervesicular aggregation through the interaction with the PMAA blocks on the hydrophilic surface of the vesicles. On the other hand, the PMAA- $b$-P(MMA-r-MAA- $r$ SpMA) copolymer produced larger vesicles in the presence of PAH at a 0.5 molar ratio of the allylamine hydrochloride (AH) unit to the SpMA unit $(D n=2.02 \mu \mathrm{m}, D w / D n=2.90)$. The vesicles had a broad size distribution and various sizes of holes in the surface. Some vesicles were coupled and bent, implying that the vesicles had the unstable bilayer. The size increase and structural destabilization of the vesicles by the electrostatic crosslinking of the hydrophobic phases agreed with the previous result that the crosslinking of the hydrophobic P(MMA-r-MAA) blocks through covalent bonding caused expansion and destabilization of the spherical vesicles [35]. By increasing the AH/SpMA ratio to 1.0 , the morphology changed into a sheet. The marks of the vesicles on the uneven and round surface of the sheet demonstrated that the sheet was formed by the fusion of the vesicles based on the combination of the hydrophobic phases via the PAH through the electrostatic interaction between the ammonium cation and sulfonate anion. As a result of further increasing the AH/SpMA ratio, the sheet morphology transformed into aggregated vesicles with a rough surface similar to the sheet surface, followed by combined vesicles with a smooth surface, and finally into spherical vesicles with a size much smaller than the original vesicles $(D n=0.773$ $\mu \mathrm{m}, D w / D n=1.42)$.

The formation of the smaller vesicles in the presence of a large excess of PAH suggested that the PAH caused the division of the vesicles. An increase in the SpMA ratio in the hydrophobic block enhanced the spherical vesicle formation. Whereas the copolymer containing 0.3 mol\% $\%$ SpMA produced the fused sheet at $\mathrm{AH} / \mathrm{SpMA}=1$, the copolymer containing 1.1-mol\% SpMA formed large round vesicles ( $D n$ $=3.00 \mu \mathrm{m}, D w / D n=1.29)$. The vesicle had a very rough surface, unlike the original vesicles. The increase in the SpMA unit in the hydrophobic phase enhanced the interaction of the units with the PAH in the individual vesicles, resulting in suppression of the intervesicular connection. However, the incorporation of more SpMA units increased the hydrophilicity of the hydrophobic phase and brought more PAH molecules into the hydrophobic phase, causing structural destabilization of the bilayer.
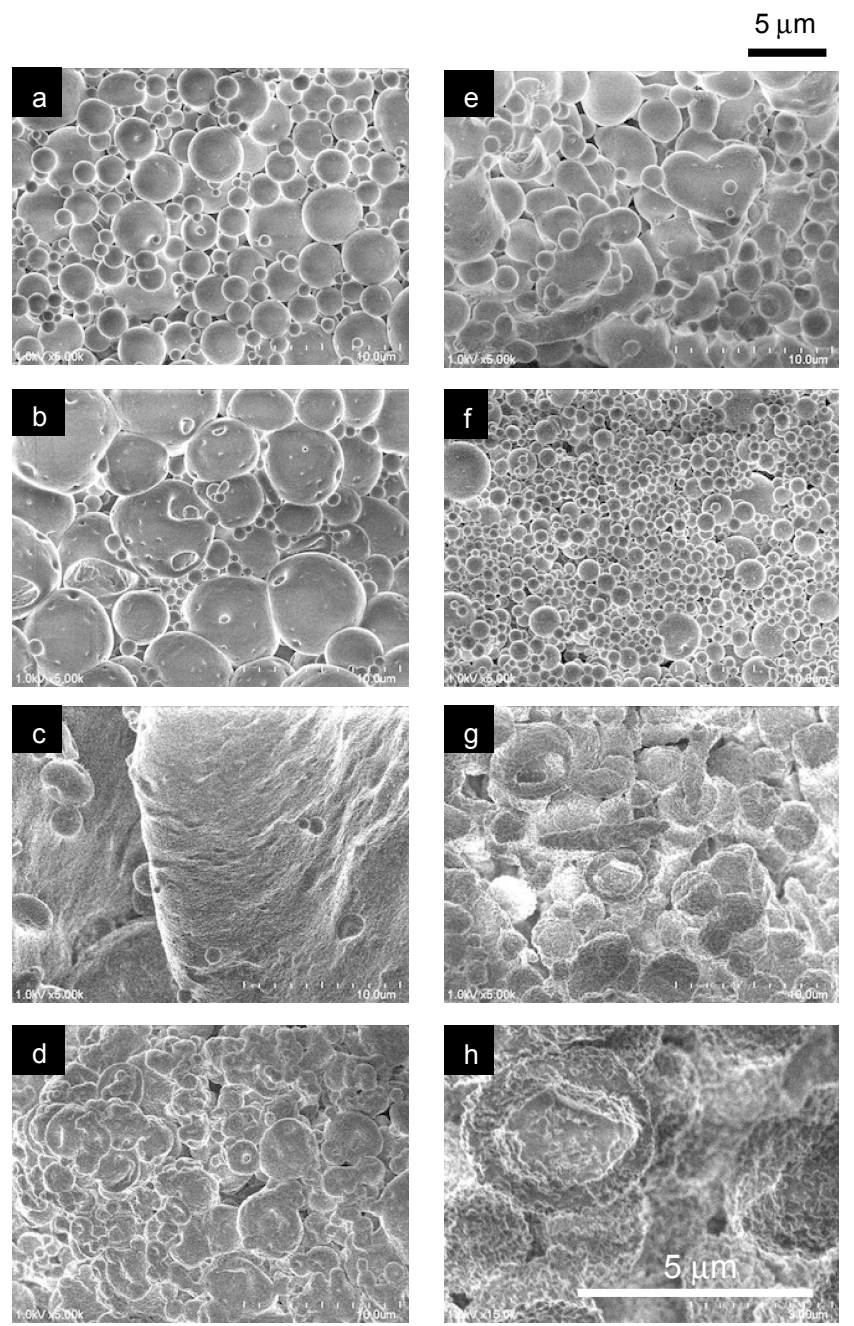

Figure 2. FE-SEM images of the morphologies formed by PMAA-b-P(MMA$r$-MAA) (a) and PMAA-b-P(MMA-r-MAA-r-SpMA) containing 0.2-0.3 mol\% SpMA $(b-h)$ in the presence of PAH $(M n=150,000)$ at $A H / S p M A=\infty(a)$, 0.5 (b), 1.0 (c), 2.5 (d), 5.0 (e), 10.0 (f), and containing $1.1 \mathrm{~mol} \%$ SpMA at $A H / S p M A=1.0$ ( $g$ and $h)$. a

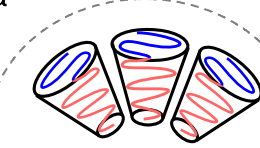

b

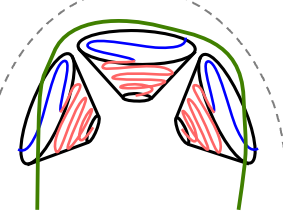

$\mathrm{PAH}$ $\sim$ PMAA block P(MMA-r-MAA) block

Figure 3. The variations in critical packing shape and curvature of the vesicles in the absence (a) and presence (b) of PAH. 

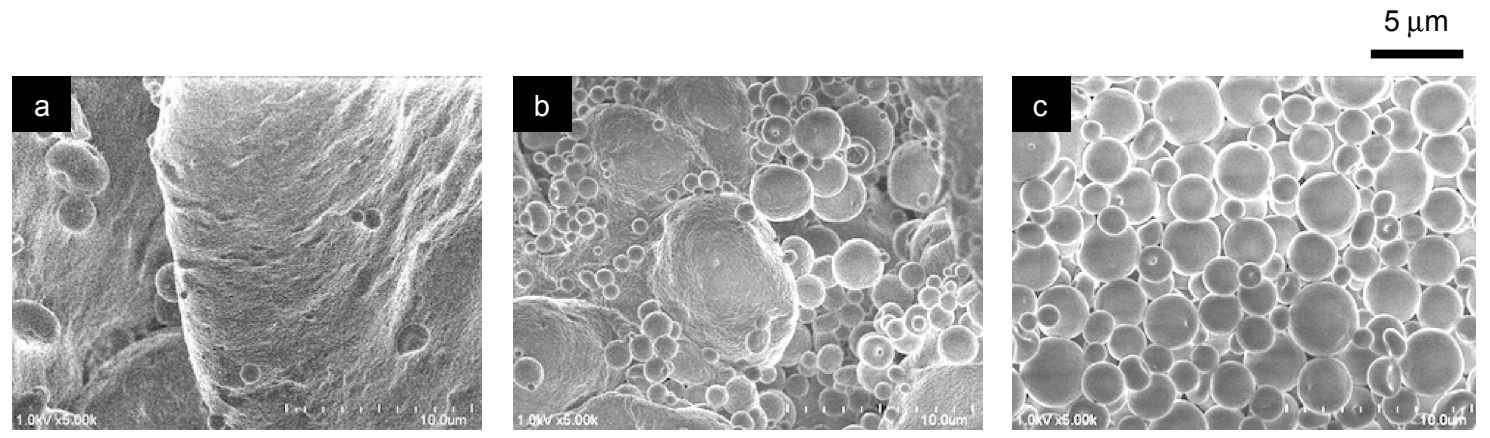

Figure 4. The morphologies of PMAA-b-P(MMA-r-MAA-r-SpMA) in the presence of $P A H$ with $M n=150,000(a), 15,000(b)$, and 3,000 (c) at AH/SpMA = 1.0.
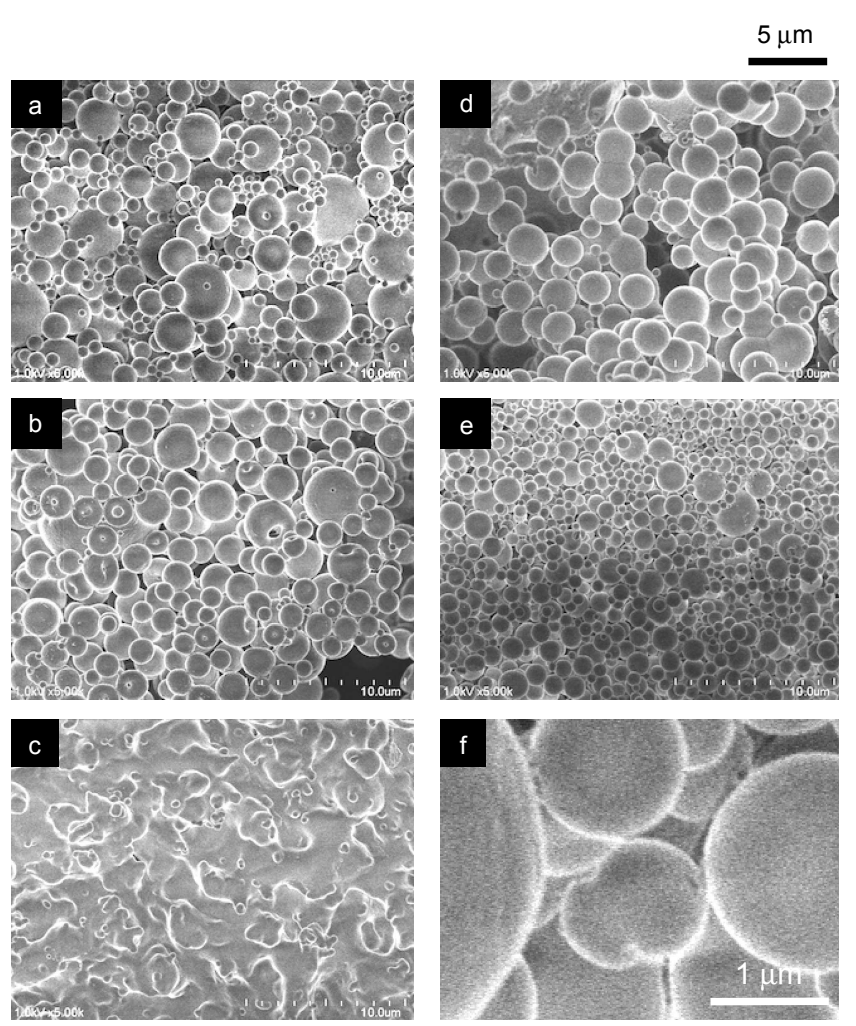

Figure 5. The variation in the morphology of $P M A A_{216}-b-P\left(M M A_{0.829}-r\right.$ $\left.M A A_{0.171}\right)_{318}(a)$ and $P M A A_{216}-b-P\left(M M A_{0.830}-r-M A A_{0.168}-r-S p M A_{0.002}\right)_{321}$ (b-f) by soaking in a solution containing $P A H(M n=150,000)$ at $A H / S p M A=\infty$ (a), 0 (b), 2.5 (c), 5.0 (d), and 10.0 (e and f).

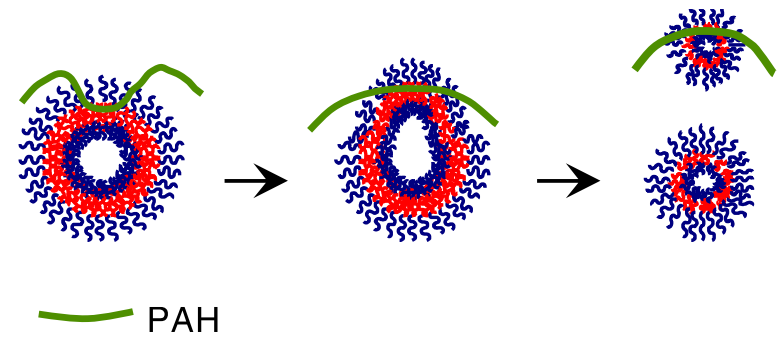

Figure 6. A schematic image for the division of the vesicles by PAH.

The molecular weight of PAH also affected the vesicle morphology. Figure 4 shows the variation in the morphology of the vesicles formed with PAH of different molecular weights. The PAH with a low molecular weight provided spherical vesicles with a smooth surface even at AH/SpMA = $1.0(D n=2.27 \mu \mathrm{m}, D w / D n=1.31)$. No connected vesicles were formed along with the spherical vesicles, indicating that the PAH had no ability to connect the hydrophobic phases of the vesicles due to its short chain.

In order to elucidate the division of the vesicles by PAH, the morphology transformation of the spherical vesicles was investigated by soaking in a PAH solution. The spherical vesicles consisting of $\mathrm{PMAA}_{216}-b-\mathrm{P}\left(\mathrm{MMA}_{0.830}-r-\mathrm{MAA}_{0.168}-r\right.$ $\left.\mathrm{SpMA}_{0.002}\right)_{321}$ with $D n=2.37 \mu \mathrm{m}$ and $D w / D n=2.12$ were soaked in an aqueous $\mathrm{MeOH}$ solution of different $\mathrm{PAH}$ concentrations. The soaking of the vesicles without SpMA $(D n=1.70 \mu \mathrm{m}, D w / D n=1.56)$ caused a slight decrease in the size and an increase in its distribution $(D n=1.12 \mu \mathrm{m}$, $D w / D n=2.71$ ). The size decrease of the SpMA-free vesicles by the soaking had a tendency similar to that for the vesicles produced by the photo NMP-induced self-assembly in the presence of PAH. The SpMA-containing vesicles were transformed into various morphologies depending on the PAH concentration (Figure 5). The vesicles were disrupted and fused at $\mathrm{AH} / \mathrm{SpMA}=2.5$. The $\mathrm{PAH}$ intervesicularly connected the hydrophobic phases by an electrostatic crosslinking between the SpMA units. The spherical shapes of the vesicles were retained at AH/SpMA $=5.0(D n=1.85$ $\mu \mathrm{m}, D w / D n=1.30$ ), while some vesicles were coupled or combined. The vesicles further changed into much smaller spherical vesicles at $\mathrm{AH} / \mathrm{SpMA}=10.0(D n=0.935 \mu \mathrm{m}$, $D w / D n=1.36)$. The observation of the coupled vesicles supports the fact that the vesicle division was caused by a large excess PAH. It is expected that the PAH was partially inserted into the hydrophobic phase from the outer hydrophilic surface of the vesicles contrary to caveolin-1 that inserts a hydrophobic phase from a cytosol, the inner hydrophilic surface because the PAH was outside of the vesicles and could not go across the hydrophobic phase. The PAH chain in the hydrophobic phase bends the bilayer outward and induces the vesicle division (Figure 6). It has been reported that the division of the SpMA-free vesicles was caused by the budding separation and invagination based on the variation in the critical packing shape of the copolymer due to growth of the hydrophobic block chain [36]. This study demonstrating the vesicle division caused by 
the electrostatic interaction with the polyelectrolyte in the hydrophobic phase can be regarded as a new artificial model for the vesicle-mediated cytosis involving a membrane protein.

\section{Conclusion}

The photo NMP-induced self-assembly for PMAA- $b$ P(MMA- $r$-MAA- $r$-SpMA) in an aqueous $\mathrm{MeOH}$ solution produced giant spherical vesicles in the absence of $\mathrm{PAH}$, whereas it provided a sheet at $\mathrm{AH} / \mathrm{SpMA}=1.0$ due to the fusion of the hydrophobic phases of the vesicles by the electrostatic interaction between the SpMA and PAH. The sheet changed into connected vesicles, followed by spherical vesicles much smaller than the original vesicles in the presence of a large excess PAH. More SpMA units in the hydrophobic block and a shorter PAH enhanced the spherical vesicle formation because the electrostatic crosslinking between the SpMA units via PAH was preferable in the individual hydrophobic phase rather than in the intervesicular hydrophobic phases. The soaking of the spherical vesicles in a PAH solution confirmed that PAH caused the division of the vesicles. It was suggested that the PAH chain in the hydrophobic phase bent the bilayer outward to divide the vesicle. This is the first study producing an artificial model of the membrane protein for the endocytosis employing the unnatural polymer vesicles and polyelectrolyte.

\section{Acknowledgements}

The author is thankful for a JSPS Grant-in-Aid for Scientific Research (Grant Number 25390003).

\section{References}

[1] H. Sprong, P. Sluijs, and G. Meer, Nature Rev. Mol. Cell Biol. 2001, 2, 504-513.

[2] B. L. Deatherage and B. T. Cookson, Infection and Immunity, 2012, 80, 1948-1957.

[3] E. J. Blott and G. M. Griffiths, Nature Rev. Mol. Cell Biol. 2002, 3, 122-131.

[4] A. Sorkin and M. Zastrow, Nature Rev. Mol. Cell Biol. 2002, $3,600-614$.

[5] S. D. Conner and S. L. Schmid, Nature, 2003, 422, 37-44.

[6] J. S. Bonifacino and B. S. Glick, Cell, 2004, 116, 153-166.

[7] H. Ewers and A. Helenius, Cold Spring Harbor Perspectives in Biology, 2011, 3, a004721, 1-14.

[8] S. Mukherjee, R. N. Ghosh, and F. R. Maxfield, Phycolog. Rev. 1997, 77, 759-803.
[9] R. G. W. Anderson, B. A. Kamen, K. G. Rothberg, and S. W. Lacey, Science, 1992, 255, 410-411

[10] C. Mineo and R. G. W. Anderson, Histochem. Cell Biol. 2001, $116,109-118$.

[11] N. J. Severs, J. Cell Sci. 1988, 90, 341-348.

[12] K. G. Rothberg, J. E. Heuser, W. C. Donzell, Y-S. Ying, J. R. Glenney, and R. G. W. Anderson, Cell, 1992, 68, 673-682.

[13] T. V. Kurzchalia, P. Dupree, R. G. Parton, R. Kellner, H. Virta, M. Lehnert, and K. Simons, J. Cell Biol. 1992, 118, 10031014.

[14] T. M. Williams and M. P. Lisanti, Genome Biol. 2004, 5, 214.1-214.8.

[15] E. Yoshida, Colloid Polym. Sci. 2013, 291, 2733-2739.

[16] E. Yoshida, Colloid Polym. Sci. 2015, 293, 649-653.

[17] E. Yoshida, Chem Xpress, 2017, 10, 118, 1-11.

[18] E. Yoshida, Colloid Polym. Sci. 2015, 293, 2437-2443.

[19] E. Yoshida, Colloid Polym. Sci. 2015, 293, 249-256.

[20] E. Yoshida, Colloid Polym. Sci. 2015, 293, 3641-3648.

[21] E. Yoshida, Colloid Polym. Sci. 2014, 292, 2555-2561.

[22] E. Yoshida, Colloid Polym. Sci. 2014, 292, 763-769.

[23] E. Yoshida, Cogent Chemistry, 2016, 2, 1212319, 1-16.

[24] E. Yoshida, Colloid Polym. Sci. 2015, 293, 1835-1840.

[25] E. Yoshida, Supramol. Chem. 2015, 27, 274-280.

[26] E. Yoshida, Open J. Polym. Chem. 2013, 3, 16-22.

[27] E. Yoshida, Colloid Polym. Sci. 2016, 294, 1857-1863.

[28] E. Yoshida, Colloid Polym. Sci. 2015, 293, 1841-1845.

[29] J. D. Mendelsohn, S. Y. Yang, J. A. Hiller, A. I. Hochbaum, M. F. Rubner, Biomacromolecules, 2003, 4, 96-106.

[30] E. Yoshida, Colloid Polym. Sci. 2010, 288, 1321-1325.

[31] E. Yoshida, Colloid Polym. Sci. 2013, 291, 993-1000.

[32] T. Miyazawa, T. Endo, S. Shiihashi, M. Ogawara, J. Org. Chem. 1985, 50, 1332-1334.

[33] E. Yoshida ISRN Polym. Sci., 2012, 102186, 1-6.

[34] S. Kobayashi, H. Uyama, I. Yamamoto, Y. Matsumoto. Polym. J. 22 (1990), 759-761.

[35] E. Yoshida, Colloid Polym. Sci. 2015, 293, 1275-1280.

[36] E. Yoshida, Colloid Polym. Sci. 2014, 292, 1463-1468. 